\title{
Elsbeere - Baum des Jahres 2011
}

Veit Martin Dörken

\begin{abstract}
Wild service tree (Sorbus torminalis) is tree of the year 2011. Its biology, ecology and use are summarized.

\section{Zusammenfassung}

Die Elsbeere (Sorbus torminalis) ist Baum des Jahres 2011. Ihre Biologie, Ökologie und ihr Nutzen werden vorgestellt.
\end{abstract}

\section{Einleitung}

Die Elsbeere (Sorbus torminalis) wurde 2011 zum 21. Baum des Jahres gekürt. Dies ist neben dem seltenen Speierling (Sorbus domestica, Jahresbaum 1993) und der für Vögel wichtigen Futterpflanze Eberesche (Sorbus aucuparia, Jahresbaum 1997) nun bereits die dritte Art aus der Gattung Sorbus. Wichtige Auswahlkriterien für die Ernennung sind z. B. Seltenheit und Bedrohung. Es gibt aber auch ästhetische, ökologische und landschaftsgestalterische Aspekte. Sorbus torminalis ist zweifelsohne eine Baum-

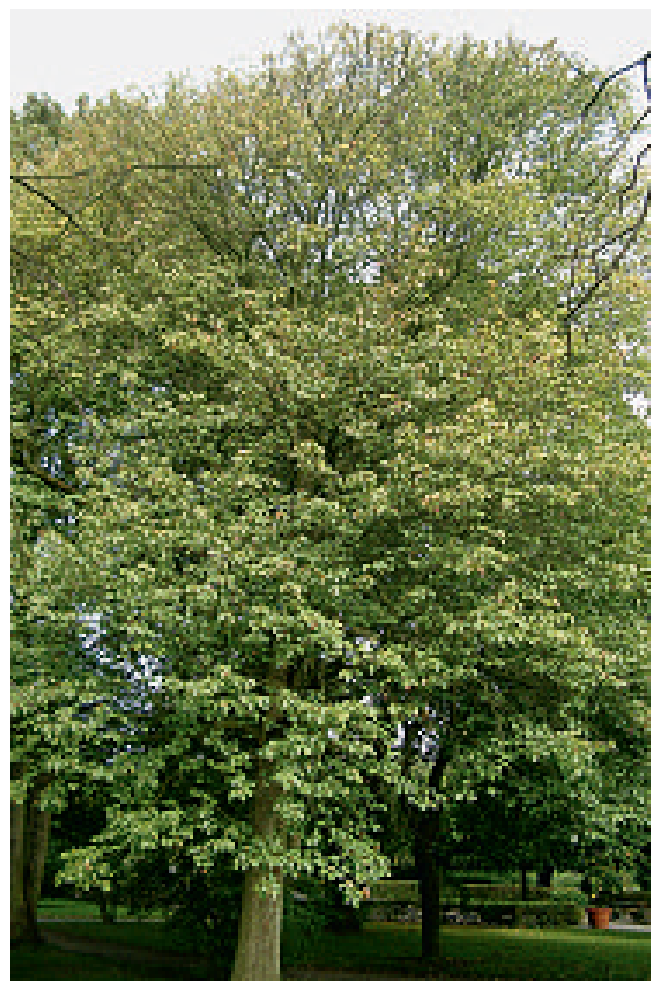

art, die der breiten Öffentlichkeit heute weitgehend unbekannt ist, obwohl sie früher als Wildobstgehölz geschätzt war. Dies war entscheidender Grund für die Ernennung zum Baum des Jahres. Weitere Auswahlkriterien waren ihre Seltenheit, das wertvolle harte Holz, die spektakuläre Herbstfärbung und ihr relativ später Blütezeitpunkt (Roloff 2011).

\section{Systematik}

Die Elsbeere gehört zur großen und vielgestaltigen Familie der Rosengewächse (Rosaceae). Sie wird zusammen mit den ebenfalls einheimischen Gattungen Amelanchier (Felsenbirne), Malus (Apfel) und Pyrus (Birne) zur Unterfamilie der Maloideae (= Apfelartigen) gestellt. Auch die Gattung Sorbus selbst ist artenreich (je nach Auffassung rund 140 Arten) und vielgestaltig. In Deutschland kommen nach der Auflistung bei Buttler \& Hand (2008) 41 heimische Sorbus-Arten vor, von denen 22 erst 2005 neu beschrieben wurden (Meyer et al. 2005). Es gibt verschiedene Ansichten zur Gliederung der Gattung. Im einfachsten Fall gibt es nur zwei Untergattungen: Aucuparia (Ebereschen mit gefiedertem Laub) und Aria (Mehlbeeren mit ungefiedertem Laub). Nach dieser Einteilung gehört S. torminalis zur Untergattung Aria (Kelly \& Hillier 2004). Andere systematische Gliederungen sind dagegen komplizierter. So unterteilt z. B. Mabberley (2008) die Gattung in vier Untergattungen, nämlich in Aria, Cormus, Torminaria und Sorbus. Meyer et al. (2005) fügen diesen noch eine fünfte Untergat-

Abb. 1: Habitus einer Elsbeere. 
tung zu (Zwerg-Mehlbeeren, Chamaemespilus). Die Elsbeere wird bei beiden zur Untergattung Torminaria gestellt. Viele Arten der Gattung sind aufgrund von Apomixis (ungeschlechtliche Samenbildung) und Hybridisierungen nur schwer $\mathrm{zu}$ bestimmen. $\mathrm{Ob}$ auch Sorbus torminalis mit anderen Arten hybridisiert, wird in der Literatur gelegentlich bezweifelt (vgl. z. B. Sснӥтt et al. 2002). Meist wird aber davon ausgegangen, dass die Elsbeere neben Sorbus aria (bzw. eng damit verwandten Arten) ein Elternteil von Sorbus $(x)$ latifolia (Breitblättrige Mehlbeere) ist (vgl. hierzu z. B. Vetvicka 1995, Wisskirchen \& Haeupler 1998).

\section{Morphologie}

Die Morphologie der Gattung Sorbus ist selbst bei den reinen Arten sehr variabel, wodurch eine eindeutige Identifizierung recht schwer fallen kann. Die Elsbeere allerdings gehört zu den wenigen Arten der Gattung, die leicht zu erkennen sind.

\subsection{Habitus}

Auf sehr schotterreichen oder sandigen, trockenen Substraten entwickelt sich die Elsbeere zu einem Großstrauch, der aufgrund zahlreicher Stockausschläge sowie Wurzelbrut Dickichte bildet. Auf nährstoffreicheren Lehm- oder Lössböden wächst sie zu einem rundkronigen Baum mit ansteigenden Ästen heran, der dann durchaus 15 bis $20 \mathrm{~m}$ hoch werden kann. Diese Höhen werden aber erst nach vielen Jahren erreicht, denn nach der raschwüchsigen Jugendphase wächst die Elsbeere später nur noch sehr langsam. Baumartige Individuen haben einen kräftigen Stamm, der in der Jugend silbrig bis grau und dicht mit Lentizellen besetzt ist. Im Alter ist die Borke dann wie beim Birnbaum (Pyrus communis) krokodilhautartig klein gefeldert und löst sich in kleinen Platten vom Stamm ab. Dadurch erhält der Stamm eine

Abb. 2 (oben): Elsbeeren haben im Gegensatz zur Vogelbeere keine Fiederblätter.

Abb. 3 (unten): Blüten der Elsbeere.
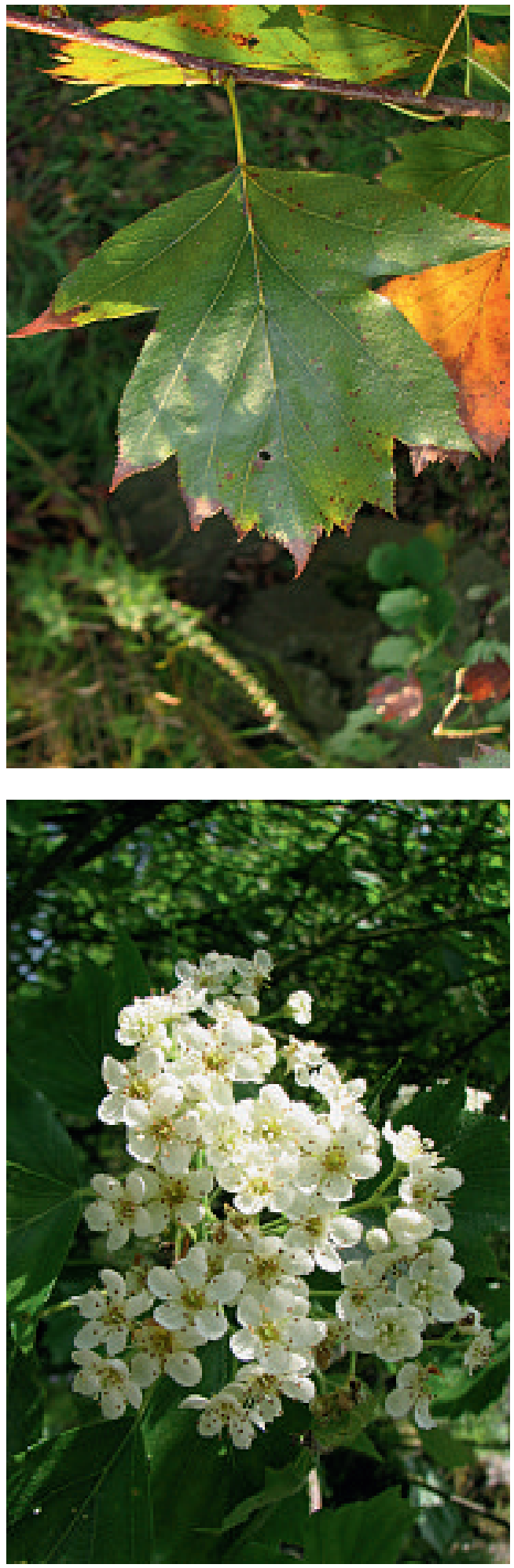

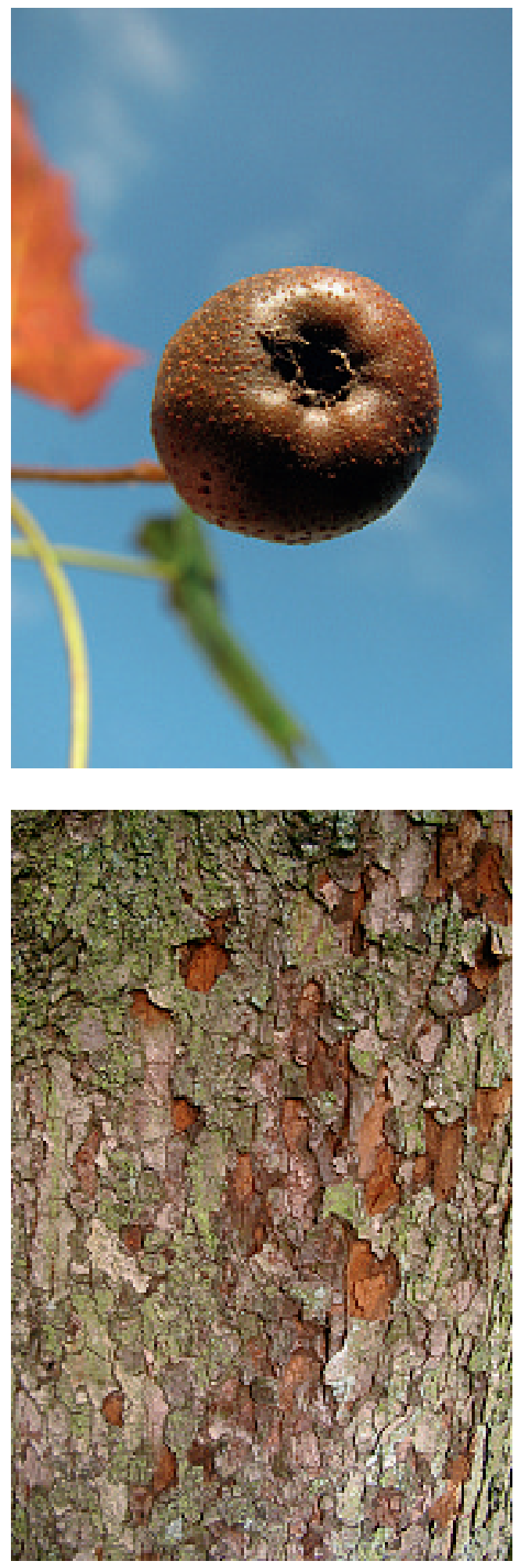

braune, beige und rote Musterung. Der Spross ist deutlich in Lang- und Kurztriebe differenziert. Junge Triebe sind zunächst locker filzig behaart und verkahlen dann rasch. Die zur Sonne exponierte Seite ist dabei meist rötlich überlaufen. Überwinterungsknospen sind in ihrer Form recht variabel: Vegetative Knospen sind länglich-eiförmig, generative Blütenknospen kugelig bis breit eiförmig. Die kahlen Knospenschuppen sind glänzend hellgrün, ähnlich denen des Berg-Ahorns (Acer pseudoplatanus), jedoch mit einem deutlichen braunen Rand. Im Gegensatz zum Ahorn stehen sie wechselständig.

\subsection{Blatt}

Sorbus torminalis ist ein winterkahles Gehölz. Die derben, leicht ledrigen, ahornartigen Blätter haben einen deutlichen Blattstiel. Die bis $15 \mathrm{~cm}$ langen Blätter sind meist tief 5 - bis 7-lappig. Die einzelnen Blattlappen laufen dabei spitz zu. Diese Blattform ist in der Gattung Sorbus nahezu einzigartig und ermöglicht daher eine leichte und sichere Identifizierung der Art. Wie auch bei den meisten triebdifferenzierten Gehölzen sind auch bei der Elsbeere die Langtriebblätter meist deutlich größer als die Kurztriebblätter. Die Blattoberseite ist glänzend dunkelgrün und kahl, die Unterseite gräulich-grün und zumindest in der Jugend weich behaart. Im Herbst färben sich die Blätter meist auffällig leuchtend goldgelb, orange bis dunkelrot.

\subsection{Blüte}

Erst im Alter von 20 bis 30 Jahren werden die ersten Blüten gebildet. Die bis $1 \mathrm{~cm}$ breiten, für die menschliche Nase streng und unangenehm duftenden Einzelblüten stehen in reichblütigen breiten Trugdolden und erscheinen recht spät von Mai bis Juni, während oder nach der Laubentfaltung. Sie ist dadurch im Vorsommer ein wichtiges Bienennährgehölz. Die Blütenstandsachsen sind dicht weißfilzig behaart. Die Blüten

Abb. 4 (oben): Apfelähnliche Frucht.

Abb. 5 (unten): Borke der Elsbeere. 
haben fünf grüne, dicht behaarte Kelchblätter und fünf in der Knospe meist dunkelrosafarbene, aufgeblüht dann weiße, kahle Kronblätter. Die meist 20 Staubblätter haben weiße Filamente und gelbe Staubbeutel. Die Blüten werden von Insekten bestäubt. Für eine erfolgreiche Befruchtung ist eine Fremdbestäubung unerlässlich, und selbst dann sterben noch rund $80 \%$ der Samenanlagen ab. Bei Selbstbestäubung reifen überhaupt keine Samen aus. So spielt Naturverjüngung über Samen zumindest vor Ort eher eine untergeordnete Rolle, während die Hauptvermehrung vegetativ über Wurzelausläufer und Stockausschläge erfolgt (Roloff 2011).

\subsection{Frucht}

Die Früchte der Elsbeere sind kugelig bis eiförmig, meist erbsengroß (nur selten über $1,5 \mathrm{~cm}$ dick) und dicht mit Korkwarzen besetzt. Sie enthalten wie Birnen zahlreiche Steinzellen. Morphologisch handelt es sich wie auch bei den Früchten des Apfelbaums (Malus domestica) um unterständige Sammelbalgfrüchte mit einem bis vier Samen. Oftmals werden sie jedoch fälschlicherweise, vielleicht wegen der geringen Größe, als Beeren bezeichnet. Aufgrund ihrer Größe und der beigen bis hellbraunen Farbe sind die Früchte im Vergleich zu anderern heimischen Sorbus-Arten recht unscheinbar.

\section{Verbreitung}

Das Areal der Gattung Sorbus erstreckt sich über die gemäßigten Breiten der gesamten Nordhemisphäre (Roloff \& BärTEls 1996). Das heutige Vielfältigkeitszentrum liegt in Europa (Meyer et al. 2005). Die Heimat der Elsbeere ist Mittel-, Ost- und Südost-Europa sowie Kleinasien und Nord-Afrika (Happe 1992). Sie ist generell ein Element der Steppengehölze und Trockenwälder und gehört zu den mediterranpontischen Florenelementen (Kiermeier 1995, Scheible 2003). Diese in Mitteleuropa seltene Art ist niemals bestandsbildend und mit deut-

Abb. 6: Zweig mit generativer Endknospe.

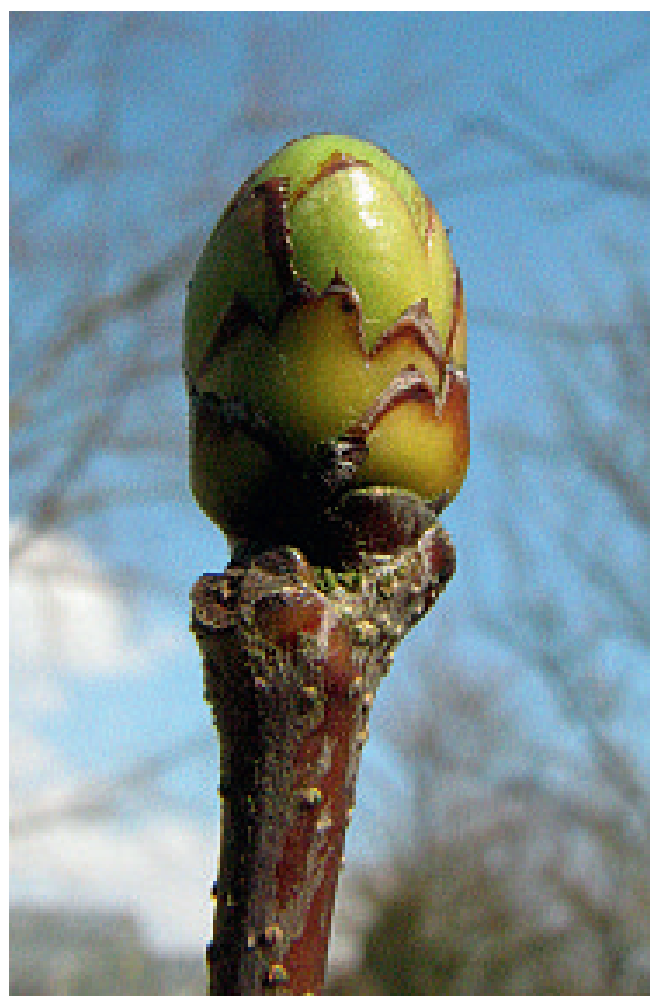

lich unter einem Prozent an der Waldfläche beteiligt (Roloff 2011).

Die Elsbeere kommt mit anderen wärmeliebenden Gehölzen wie z. B. Flaum-Eiche (Quercus pubescens), Winter-Linde (Tilia cordata), Liguster (Ligustrum vulgare), Echtem Kreuzdorn (Rhamnus catharticus) und Wolligem Schneeball (Viburnum lantana) vor. Elsbeeren sind auch an Wegrändern, Böschungen, Fels- und Trockenhängen und in Feldgehölzen zu finden. Bevorzugt werden nährstoffreiche, gut drainierte, basenreiche Substrate besiedelt. Die Art fehlt auf nährstoffarmen, sehr sauren Sanden oder auf zu schweren staunassen Tonböden. In generell wärmeren Gebieten kommt die Elsbeere auch an Standorten vor, deren Oberboden eine leicht saure Bodenreaktion zeigt, vorausgesetzt, die Substrate sind entsprechend nährstoffreich. Nach HAPPE (1992) besiedelt $S$. torminalis die trocken-warmen, basischen Standorte nicht aufgrund ihrer Vorliebe für solche Standorte, sondern weil sie wegen ihrer geringen Konkurrenzfähigkeit auf diese Bereiche 


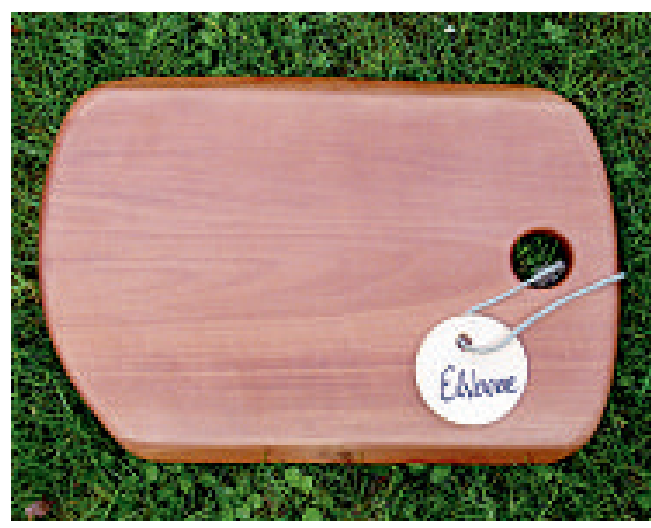

verdrängt wird. Hier ist sie wegen ihrer hohen Trockenheitsverträglichkeit anderen Gehölzen deutlich überlegen. Die Nordwestgrenze der mitteleuropäischen Verbreitung verläuft in Deutschland quer durch Nordrhein-Westfalen. Elsbeeren stellen typische Zeiger für ein warmes Lokalklima dar.

\section{Verwendung}

Die Elsbeere ist ein Wildobstgehölz, dessen Früchte reich an Vitamin C sind (DülL \& KütZelnigg 2005). Vor Frösten schmecken die Früchte mehlig. Es bildet sich nach dem Verzehr ein unangenehmes pelziges Gefühl auf der Zunge, ähnlich wie nach dem Essen von Schlehen (Prunus spinosa). Erst nach Frosteinwirkung schmecken Elsbeeren-Früchte saftig süß-sauer. Sie eignen sich zur Herstellung von Marmelade und hochwertigen Likören. Bereits im antiken Rom wurden die gerbstoffreichen Fruchtsäfte medizinisch gegen Ruhr verabreicht, daher auch der botanische Artbeiname torminalis (von lat. tormina = Bauchschmerzen, Düll \& Kutzelnigg 2005). Woher die Bezeichnung Elsbeere stammt, ist heute nicht mehr festzustellen (Happe 1992).

Die Früchte eigen sich außerdem zur Herstellung von Trockenobst und zur Zubereitung von Tee (Fleischhauer et al. 2007). Neben den Früchten liefert die Elsbeere ein wertvolles, zähes Hartholz, welches im Handel unter der Bezeichnung Schweizer Birnbaum geführt wird (Sснüтt et al. 2002). Es eignet sich für Schnitzereien, wird aber auch zur Herstellung von
Zollstöcken und Werkzeugteilen genutzt (Höll 1983). Zudem werden aus dem Holz hochwertige Furniere hergestellt. Ansonsten ist die Elsbeere in ländlichen Regionen ein idealer Hof- und Dorfbaum. Aufgrund ihrer hübschen Blüten und der auffälligen Herbstfärbung ist die Elsbeere ein geeignetes Solitärgehölz für Parkanlagen. Ein reicher Fruchtbesatz zeichnet die Elsbeere zudem als ideales Nährgehölz für Vögel aus. Aufgrund ihrer vielen positiven Eigenschaften und ihrer Resistenz gegenüber heißen, trockenen Sommern sollte die Elsbeere in Zukunft wieder mehr Beachtung finden und häufiger gepflanzt werden.

\section{Literatur}

Buttler, K. P. \& Hand, R. 2008: Liste der Gefäßpflanzen Deutschlands. - Kochia, Beih. 1.

Düll, R. \& Kutzelnigg, H. 2005: Taschenlexikon der Pflanzen Deutschlands. Ein botanisch-ökologischer Exkursionsbegleiter. 6. Aufl. - Wiebelsheim.

Fleischhauer, S. G., Guthmann, J. \& Spiegelberger, R. 2007: Essbare Wildpflanzen. - Baden, München. Happe, J. 1992: Verbreitung der Elsbeere (Sorbus torminalis) im forstlichen Wuchsgebiet Weserbergland in Nordrhein-Westfalen. - Ber. Naturwiss. Ver. Bielefeld Umgeg. 33: 145-172.

HöLl, W. 1983: Bäume in Mitteleuropa. - Köln.

Kelly, J. \& Hillier, J. 2004: The Hillier. Bäume und Sträucher. - Braunschweig.

Kiermeier, P. 1993: Wildgehölze des mitteleuropäischen Raumes, BdB-Handbuch, Teil VIII, 5. Aufl. - Pinneberg. Kiermeier, P. 1995: Lebensbereiche der Gehölze, eingeteilt nach dem Kennziffersystem, 3. Aufl. Pinneberg.

Mabbrley, D. J. 2008: Mabberley's plant book, 3. Aufl. - Cambridge.

Meyer, N., Meierott, L., Schwerk, H. \& Angerer, O. 2005: Beiträge zur Gattung Sorbus in Bayern. - Ber. Bayer. Bot. Ges., Sonderb.: 5-216.

Roloff, A. 2011: Baum des Jahres 2011: Die Elsbeere. - Ginkgoblätter, Mitt. Dtsch. Dendrol. Ges. 121: 30-31. Roloff, A. \& B̈̈rtels, A. 1996: Gartenflora. Band 1: Gehölze. - Stuttgart.

Scheible, A. 2003: Die Elsbeere in NRW, Bestand und Entwicklung von 1992-2002. - LÖBF-Mitt. 28: 44-47. Sснӥтt, P., Sснuск, H. J. \& Sтімm, B. 2002: Lexikon der Baum- und Straucharten. - Hamburg.

Vetvicka, v. 1995: Dausien’s großes Buch der Bäume und Sträucher. - Hanau.

Wisskirchen, R. \& Haeupler, H. 1998: Standardliste der Farn- und Blütenpflanzen Deutschlands. - Stuttgart.

Abb. 7: Küchenbrett aus Elsbeerenholz. 